\title{
Fisheye Lens for Image Processing Applications
}

\author{
Gyeong-il Kweon* \\ Department of Optoelectronics, Honam University \\ 59-1, Seobong-dong, Gwangsan-gu, Gwangju, 506-714, Republic of Korea \\ Young-ho Choi \\ Department of Information and Communications Engineering, Honam University \\ 59-1, Seobong-dong, Gwangsan-gu, Gwangju, 506-714, Republic of Korea \\ Milton Laikin \\ Laikin Optical Corporation \\ 4314 Marina City Drive \#930, Marina Del Rey, CA 90292, USA
}

(Received May 26, 2008 : accepted June 12, 2008)

\begin{abstract}
We have developed a miniature fisheye lens with $190^{\circ}$ field of view operating simultaneously in the visible and the near infrared wavelengths. The modulation transfer function characteristic for the visible wavelength is sufficient for a mega-pixel-grade image sensor. The lens also has a fair resolution in the infrared wavelength region. The calibrated $\mathrm{f}-\Theta$ distortion is less than $5 \%$, and the relative illumination is over $90 \%$. In consequence, a sharp wide-angle image can be obtained which is uniform in brightness over the entire range of field angles. The real image heights for the visible and the near infrared wavelengths have been fitted to polynomial functions of incidence angle with sub-pixel accuracies. Combined with the near equidistance projection scheme of the lens, this lens can be advantageously employed in various image-processing applications requiring a wide-angle lens.
\end{abstract}

Keyword: fisheye lens, image processing, equidistance, lens design

OCIS codes : (080.3620) Lens design ; (100.2000) Digital image processing ; (220.0220) Optical design and fabrication

\section{INTRODUCTION}

Fisheye lenses have a long history of development [1-14]. Their enormous fields of view (FOV) combined with the unavoidable large distortion lead many investigators to seek a means of extracting visually more pleasing images or computationally more convenient images from raw fisheye images. Image processing of fisheye images have been used in various disciplines including robotics [15-16], security and surveillance [1719], and entertainment [20-21]. Obtaining panoramic images from fisheye images has been particularly popular among photographers as well as others. However, the

*Corresponding author: kweon@honam.ac.kr employed image processing algorithms have been inaccurate. The main cause of inaccuracy has been the poorly defined notion of panorama, and the crude modeling of the image formation process in the fisheye lens.

Fisheye lens based image processing has also been used for virtual touring. In this application, two fisheye lenses each separately having a FOV larger than $180^{\circ}$ are employed in back-to-back configuration. Since each fisheye lens captures the view of a hemisphere, two lenses as a whole capture the images of an entire sphere. Then, a perspectively normal image can be extracted from the combined image. For such applications, it is critical that the FOV of the fisheye lens be larger than $180^{\circ}$, and the projection scheme of the lens be close to an 


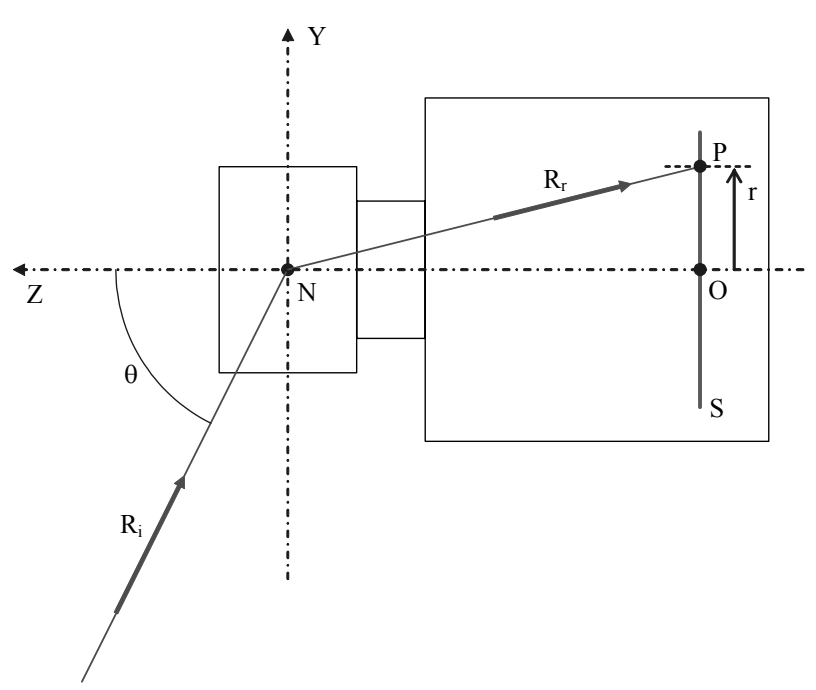

FIG. 1. Schematic diagram illustrating the projection scheme of a general wide-angle lens.

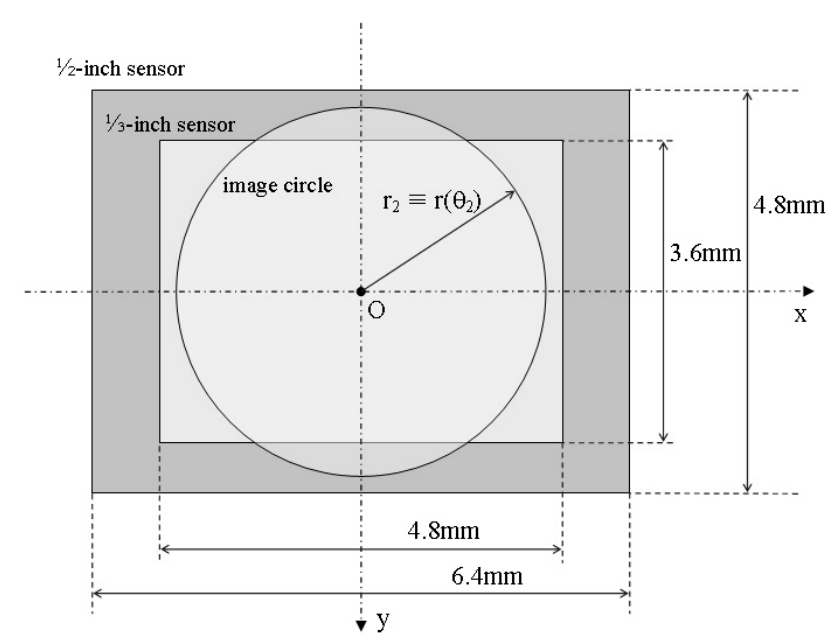

FIG. 2. Typical image sensor formats and the desirable image size of the fisheye lens under investigation.

equidistance projection scheme.

Figure 1 is a schematic diagram illustrating the projection scheme of a general wide-angle lens. The optical axis coincides with the Z-axis of the coordinate system, and the incidence angle $\theta$ of an incident ray $R_{\mathrm{i}}$ is measured as a zenith angle. All the rays forming image points on the image sensor plane $\mathrm{S}$ are considered to pass through the nodal point $\mathrm{N}$ of the lens. The intersection between the optical axis and the image sensor plane $\mathrm{S}$ is designated as origin $\mathrm{O}$ in Fig. 1. The refracted ray $R_{r}$ corresponds to the incident ray $R_{i}$, and forms an image point $\mathrm{P}$ on the image sensor plane $\mathrm{S}$. The radial distance from the origin $\mathrm{O}$ to the image point $\mathrm{P}$ is the image height $r$.

The general projection scheme of a lens can be defined as $r=r(\theta)$, where the image height $r$ is a monotonically increasing function of the incidence angle $\theta$. For fisheye lenses, equidistance projection schemes are the most popular projection schemes. If the maximum incidence angle is $\theta_{2}$, and the corresponding maximum image height is given as $r_{2}$, then an equidistance projection scheme can be given as

$$
r_{e d}(\theta)=\frac{r_{2}}{\theta_{2}} \theta,
$$

where $r_{e d}$ is the image height following an equidistance projection scheme. Then the distortion from the equidistance projection scheme can be defined as

$$
\operatorname{distortion}(\theta)=\frac{r_{e d}(\theta)-r_{r p}(\theta)}{r_{e d}(\theta)} 100
$$

where $r_{r p}(\theta)$ is the real image height, which can be experimentally measured using an actual (i.e., physically realized) lens.

To apply an image processing algorithm to images obtained using a fisheye lens, the projection scheme of the lens must be known accurately. Provided a complete lens prescription is known, the projection scheme can be theoretically calculated using a dedicated lens design program such as Code V or Zemax. Since lens manufactures do not normally reveal the lens structure they are offering for sale, researchers have been experimentally measuring the projection schemes of the lens as well as the location of the optical axis in the image (i.e., image center). The experimental determination of the above parameters is a project of its own and considerable literature exists in the fields [22-23]. Furthermore, the experimental methods are highly susceptible to errors and are painstaking.

The purpose of the present article is to provide a miniature fisheye lens with FOV larger than $180^{\circ}$ and which has sufficient resolution for a mega-pixel-grade image sensor. Since this lens is particularly targeted for image processing applications, it is intended that the real projection scheme of the lens is provided in as much detail as possible so that the potential investigators using the developed lens need not measure the projection schemes by themselves, thus saving themselves a lot of turmoil.

\section{LENS DESIGN}

Figure 2 shows the most popular image sensor formats and the desired image size for the fisheye lens under investigation. Most of the image sensors have rectangular shapes with width $\mathrm{W}$ and height $\mathrm{H}$ having the ratio of $4: 3$. For a $1 / 2$-inch image sensor, the width is $6.4 \mathrm{~mm}$, and the height is $4.8 \mathrm{~mm}$. On the other hand, for a $1 / 3$-inch image sensor, the width is $4.8 \mathrm{~mm}$, while 


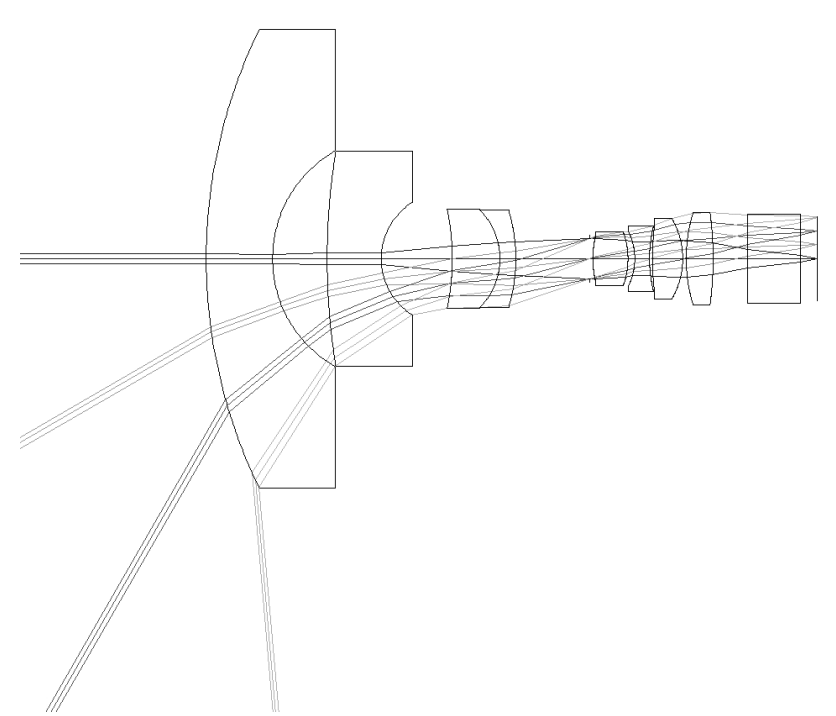

FIG. 3. Optical layout and the ray trajectories for the designed fisheye lens.

the height is $3.6 \mathrm{~mm}$. To obtain more than a hemispherical image using a single fisheye lens, the maximum incidence angle $\theta_{2}$ has been chosen as $90^{\circ}$, and the corresponding image height $r_{2}$ has been chosen as 2.35 mm. This particular choice of figure has been taken so that $180^{\circ}$ horizontal FOV is obtained using a $1 / 3$-inch image sensor, while full circle fisheye image can be obtained using a 1/2-inch image sensor.

Figure 3 shows the optical layout of the designed fisheye lens. To keep the number of lens elements low, high index optical glasses were required for the first few lens elements. However, the optical glasses with refractive index higher than 1.8 has been avoided due to its high material cost and difficulty in grinding and polishing. The first two lens elements are actually made of the same glass from Hikari optics corp., namely E-LASF016. This particular glass has a relatively high refractive index of 1.7724, and a moderate Abbe number of 49.61 . The mechanical properties of this glass are well known and appropriate for mass production of precision optical parts. The next two lens elements form a cemented optical doublet in order to reduce chromatic aberration. After a fixed aperture optical stop, four more lens elements are followed to form nearly telecentric converging rays toward the image sensor plane. The back focal length of the lens is forced to agree with a typical number for board lenses with Hitachi M12 mount. Particular attention has been paid to avoid a lens element with a shape which is difficult to manufacture. The desirable

TABLE 1. Lens prescription for the designed fisheye lens.

\begin{tabular}{|c|c|c|c|c|c|c|}
\hline Surface no. & Comment & Radius & Thickness & $\begin{array}{c}\text { Refractive } \\
\text { index }\end{array}$ & $\begin{array}{c}\text { Abbe } \\
\text { number }\end{array}$ & Glass \\
\hline Obj & & $\infty$ & $\infty$ & & & \\
\hline 1 & Lens 1 & 29.794 & 3.801 & 1.7724 & 49.61 & E-LASF016 \\
\hline 2 & & 7.112 & 3.120 & & & \\
\hline 3 & Lens 2 & 37.956 & 3.089 & 1.7724 & 49.61 & E-LASF016 \\
\hline 4 & & 3.798 & 4.071 & & & \\
\hline 5 & Lens 3 & -13.824 & 2.725 & 1.7616 & 26.56 & E-SF14 \\
\hline 6 & Lens 4 & -3.990 & 0.911 & 1.6967 & 55.53 & E-LAK14 \\
\hline 7 & & -9.399 & 4.205 & & & \\
\hline Stop & Stop & $\infty$ & 0.200 & & & \\
\hline 9 & Lens 5 & 5.358 & 2.011 & 1.5687 & 56.34 & E-BAK4 \\
\hline 10 & & -4.515 & 0.390 & & & \\
\hline 11 & Lens 6 & -3.527 & 0.842 & 1.8049 & 25.43 & E-SF6 \\
\hline 12 & & 7.737 & 0.169 & & & \\
\hline 13 & Lens 7 & 15.055 & 1.731 & 1.6203 & 60.29 & E-SK16 \\
\hline 14 & & -4.574 & 0.200 & & & \\
\hline 15 & Lens 8 & 8.965 & 1.539 & 1.6399 & 60.09 & E-LAK01 \\
\hline 16 & & -20.109 & 1.986 & & & \\
\hline 17 & OLPF & $\infty$ & 3.000 & 1.5167 & 64.10 & E-BK7 \\
\hline 18 & & $\infty$ & 1.000 & & & \\
\hline IMA & CMOS & $\infty$ & & & & \\
\hline
\end{tabular}




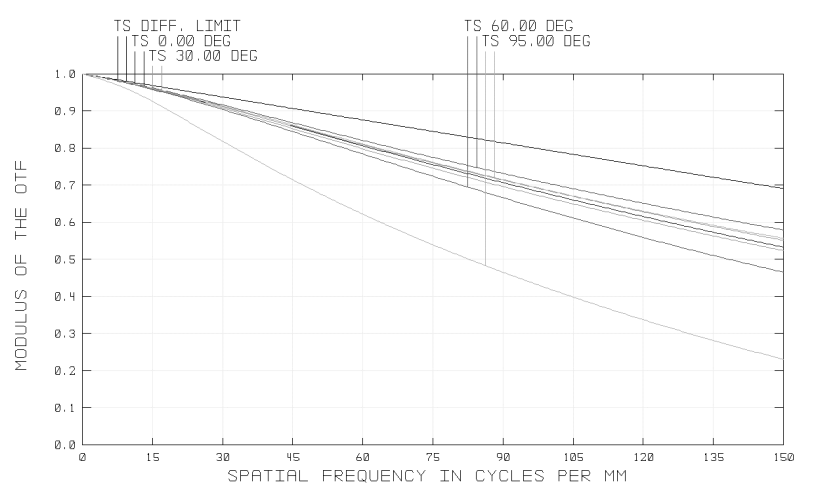

FIG. 4. Modulation transfer function characteristics of the lens in the visible wavelength region.

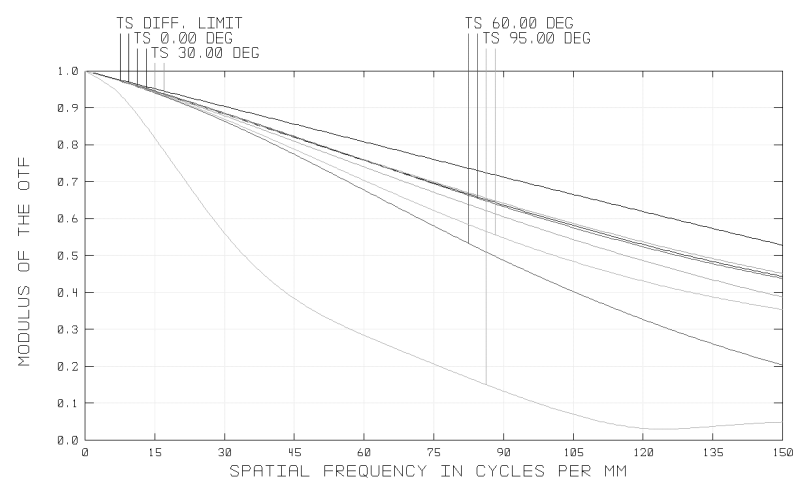

FIG. 5. : Modulation transfer function characteristics of the lens in the near infrared wavelength region.

projection scheme given Eq. (1) has been entered into the merit function operand REAY in Zemax. Another difficult part was ensuring standard manufacturing tolerances while keeping the overall length not excessively long. The optimized design shown in Fig. 3 has tolerances which can be routinely met by the skilled lens makers. The FOV of the optimized lens is $190^{\circ}$ with a F-number of 2.8. The complete lens prescription is given in table 1.

Figure 4 shows the modulation transfer function (MTF) characteristic of the lens for the visible wavelength. It can be seen that the MTF is over 0.2 at 150 $\mathrm{lp} / \mathrm{mm}$ for all field angles. Excluding the edges of the images having the full field angle, it is expected that the lens is suitable for multi-mega-pixel-grade image sensor. Figure 5 shows the MTF characteristic for the near infrared wavelength $(0.85 \sim 0.94 \mu \mathrm{m})$. These particular wavelengths correspond to the most popular center wavelengths of infrared LED illuminators used in security industry. Although the MTF is lower than that for the visible wavelength, still it is good enough for D1 grade image sensor.

Figure 6 shows the calibrated $\mathrm{f}-\Theta$ distortion in the visible wavelength range. This is practically the same as the distortion defined in Eq. (2). As can be seen
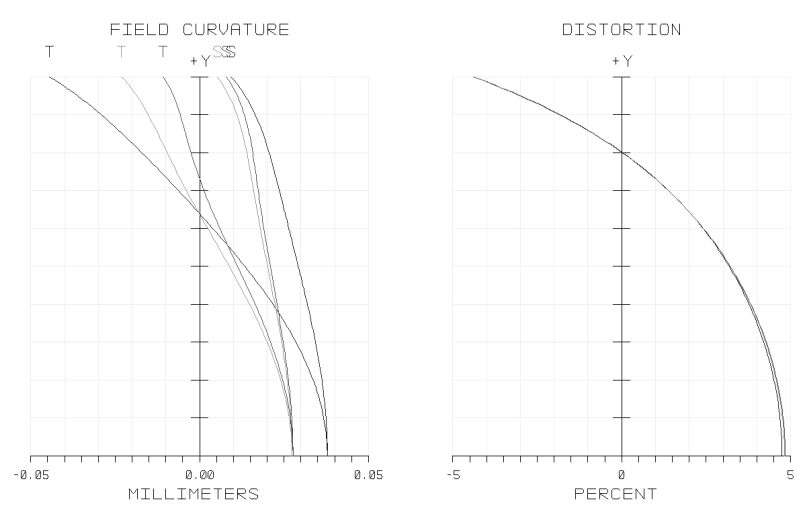

FIG. 6. Field curvature and the distortion in the visible wavelength region.

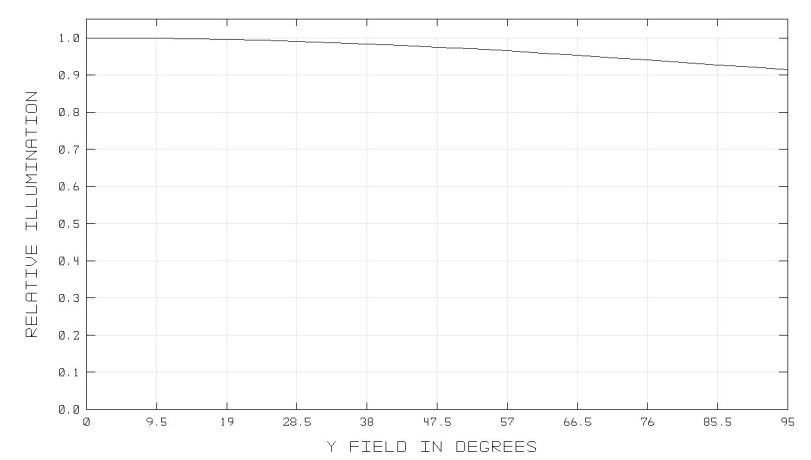

FIG. 7. Relative illumination in the visible wavelength region.

from the figure, the distortion is less than $5 \%$ even at the full field. Considering the FOV of this lens, this figure is considered fair. Figure 7 shows the relative illumination in the visible wavelength range, and it can be seen that the relative illumination at the full field is more than $90 \%$ of that at the zero field. Therefore, the resulting image will be very uniform in brightness. The relative illumination for the near infrared wavelength is comparable to this.

Figure 8 shows the physical dimension of the developed lens. The overall length from the first surface to the image sensor is $35.0 \mathrm{~mm}$, and the back focal length is $5.76 \mathrm{~mm}$ taking into account a $3 \mathrm{~mm}$ thick E-BK7 optical low pass filter (OLPF). The diameter of the lens from the front side is $30.6 \mathrm{~mm}$. This lens is designed as a board lens and has a Hitachi M12 mount. Barrel and spacers are carefully designed so that it neither occludes any ray within the valid range of field angles nor allows any ray outside the range reach the image sensor. Figure 9 shows a cut-away perspective view of the lens, and Fig. 10 is a photograph of a developed fisheye lens.

Figure 11 shows the fisheye lens mounted on a $3 \mathrm{M}$ pixel CMOS camera (CMOS Uroria from ViewBits Inc.). The optical axis has been aligned vertical to the 


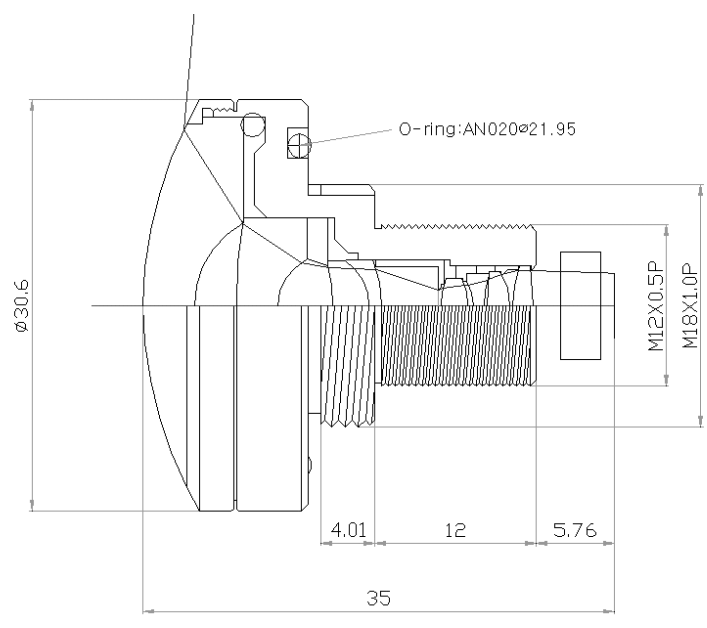

FIG. 8. Mechanical structure of the fisheye lens.

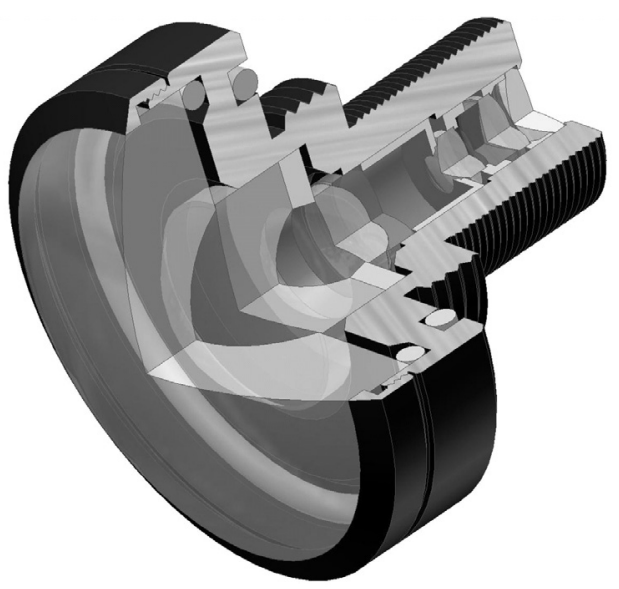

FIG. 9. Perspective view of the fisheye lens.

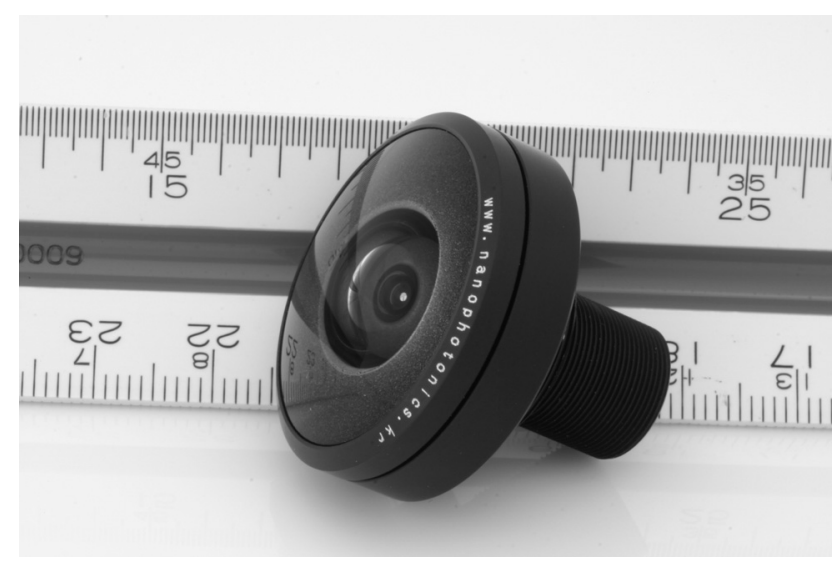

FIG. 10. A photograph of the developed fisheye lens.

ground using a two-axis electronic level meter. Figure 12 shows the view of the Honam university from a plaza between the university library and the student cafeteria. Figure 13 is a panoramic image obtained from Fig. 12 applying a simple polar-to-rectangular transformation. From Fig. 13, it is obvious that the FOV of

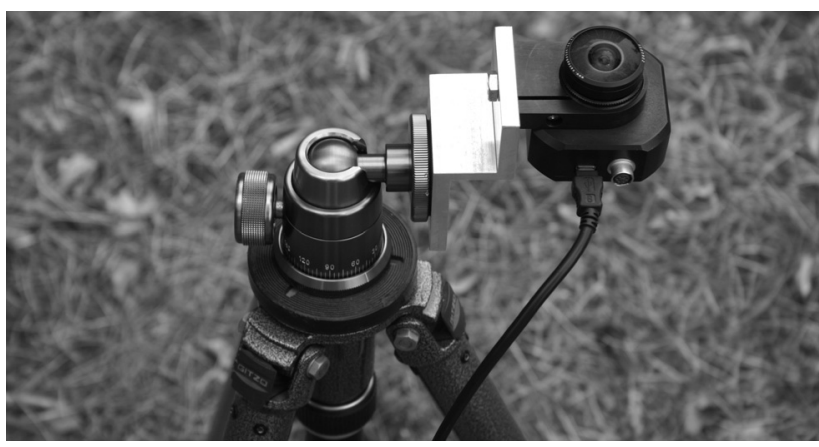

FIG. 11. An experimental setup to take a whole sky view using the developed fisheye lens.

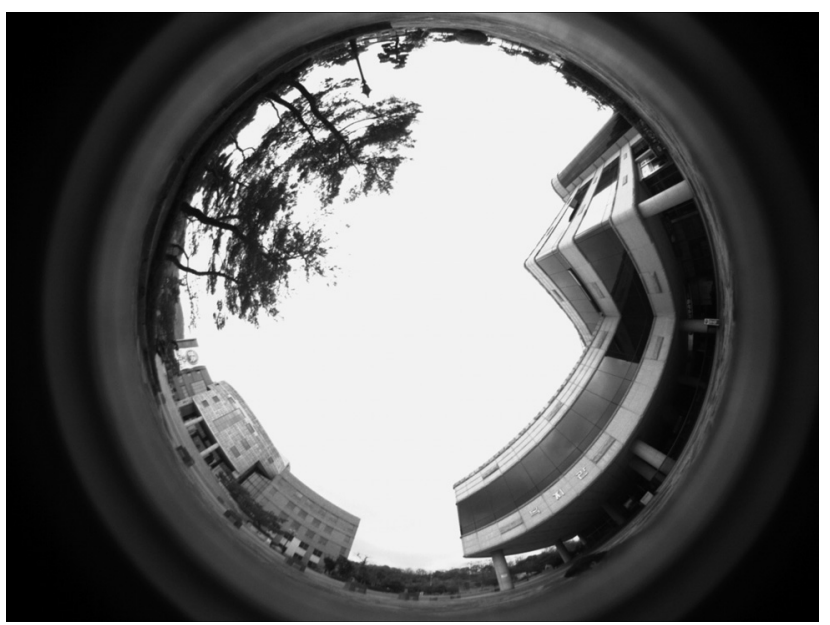

FIG. 12. A whole sky view obtained using the developed fisheye lens mounted on a $3 \mathrm{M}$ pixel CMOS camera.

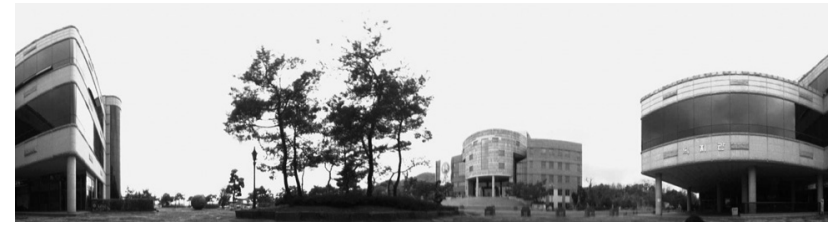

FIG. 13. Sample image obtained applying a simple polarto-rectangular transformation to the photograph in Fig. 12.

the lens is at least larger than $180^{\circ}$ since objects below the horizon are captured all around the camera.

Figure 14 is a sample image of an office interior obtained with the optical axis aligned parallel to the ground. Figure 15 shows details of the image from the leftmost edge of the photograph. From the figure, it can be seen that the resolution of the image is limited by the grainess of the pixel from the CMOS image sensor, and not by the resolution of the fisheye lens. Therefore, it can be concluded that the developed fisheye lens has enough resolution for a mega-pixel grade image sensor. 


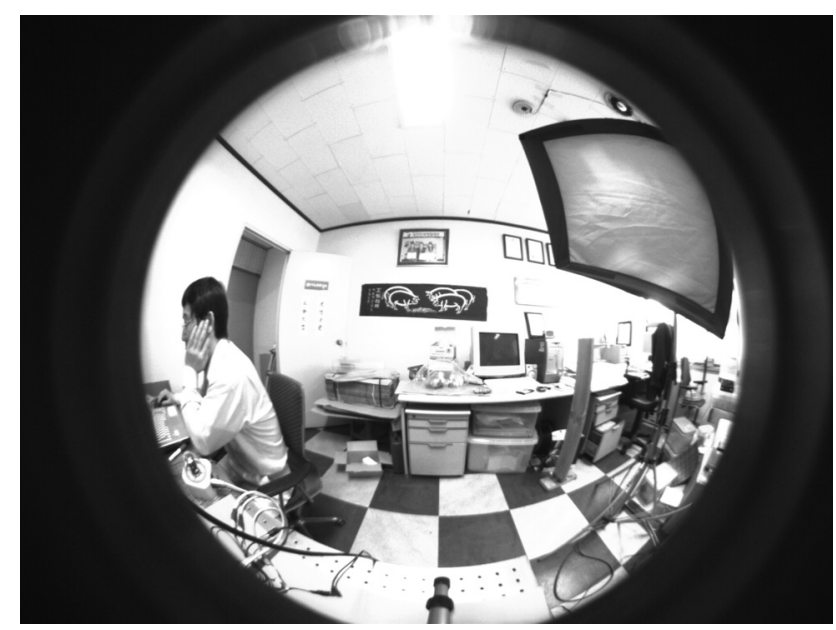

FIG. 14. A sample image of an office interior obtained using the developed fisheye lens.

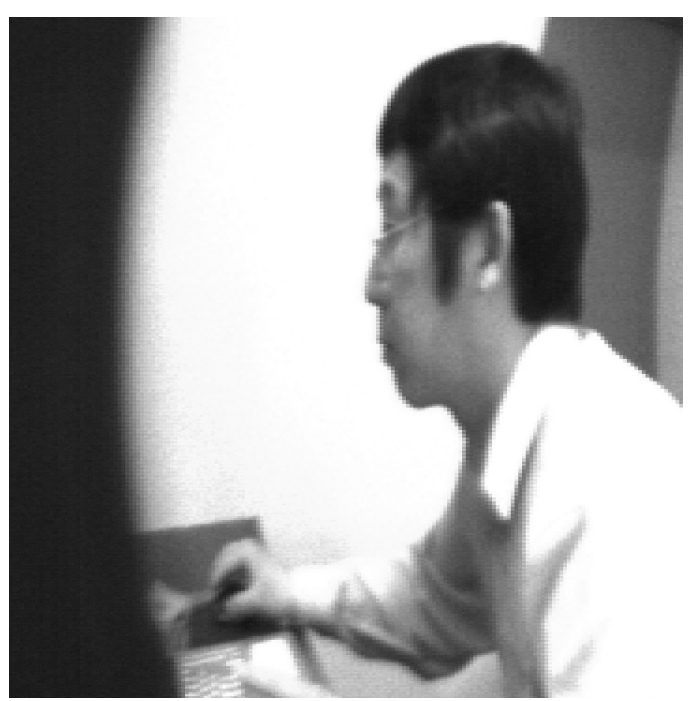

FIG. 15. Details of Fig. 14 at the left edge.

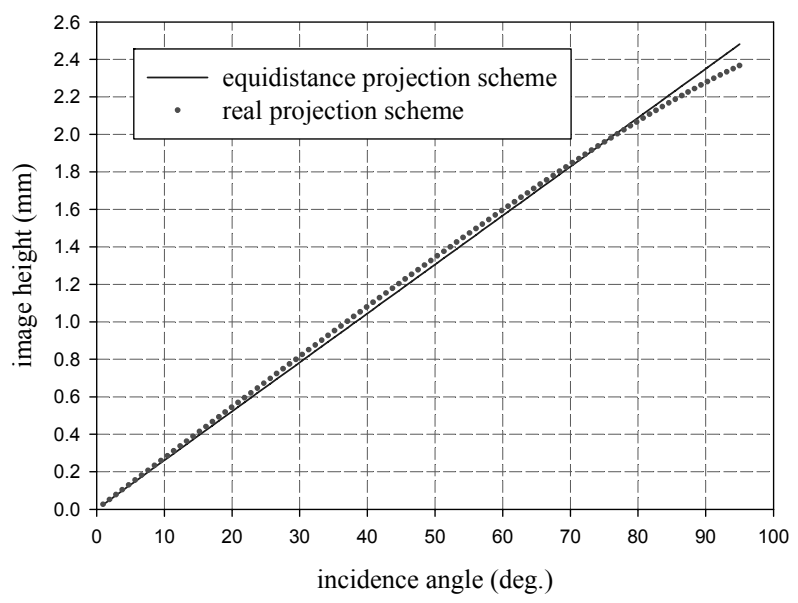

FIG. 16. Comparison of the real projection scheme of the lens to an ideal equidistance projection scheme.

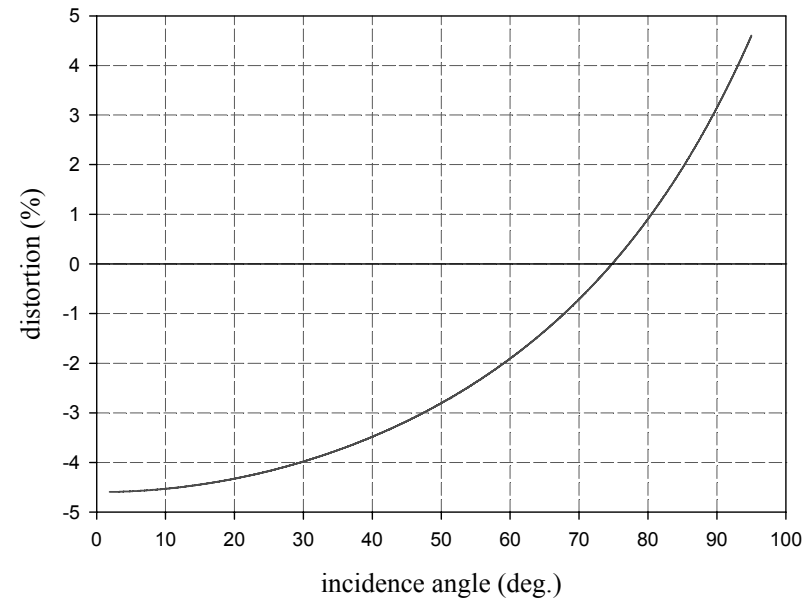

FIG. 17. Distortion of the lens as a percentage error from an ideal equidistance projection scheme.

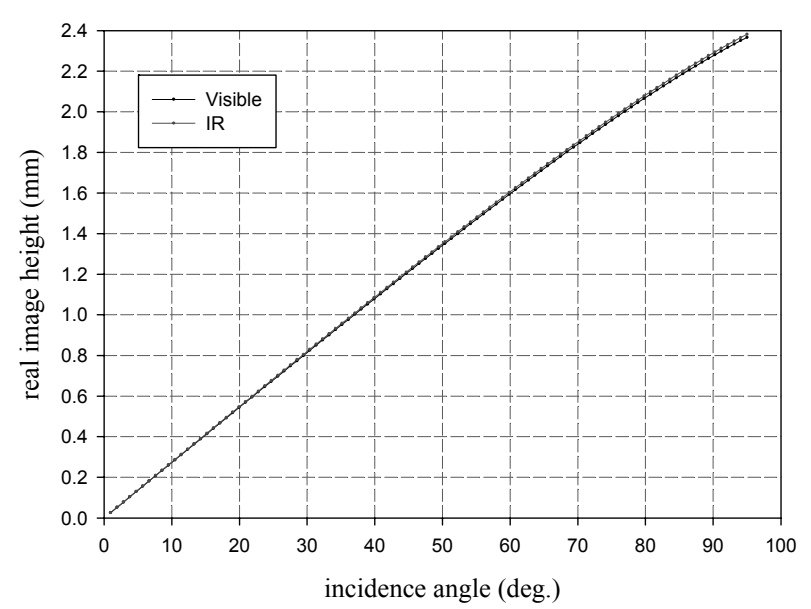

FIG. 18. Comparison of the real projection schemes for the visible and the near infrared wavelengths.

\section{REAL PROJECTION SCHEME}

As has been said earlier, the real projection of a lens should be known accurately in order to be used in image processing applications. Figure 16 shows the ideal equidistance projection scheme (solid line) described by Eq. (1) and the real projection scheme (dotted line) of the lens obtained using a merit function operand REAY. Figure 17 is the distortion calculated using Eq. (2), and the distortion is less than $5 \%$ over the entire field angles.

Figure 18 shows the real projection scheme in the visible wavelength range (solid line) and that in the near infrared wavelength range (dotted line). As can be seen, the discrepancy is not large, but the discrepancy increases as the field angle increases. Figure 19 shows the discrepancy between the two real projection schemes. At the full field, the discrepancy is about $15 \mu \mathrm{m}$. 


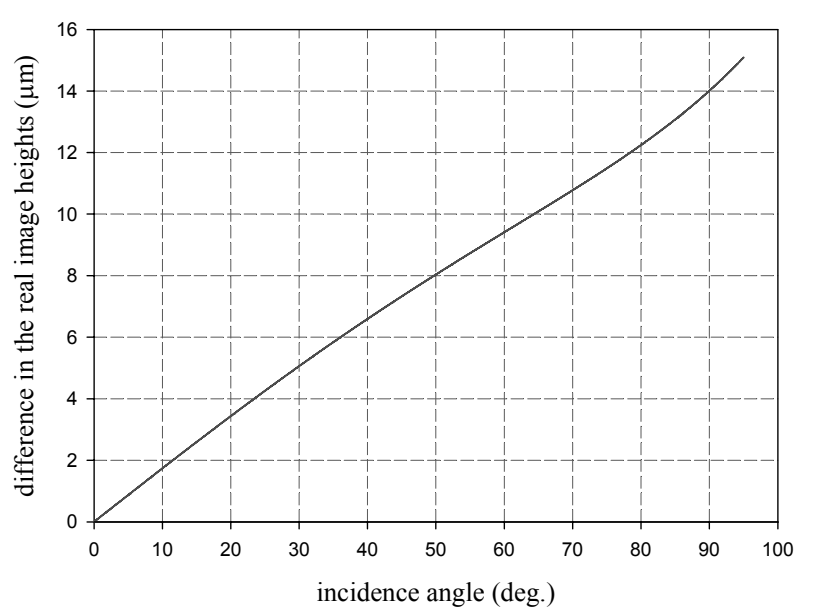

FIG. 19. Deviation of the real image height for the near infrared wavelength from that for the visible wavelength.

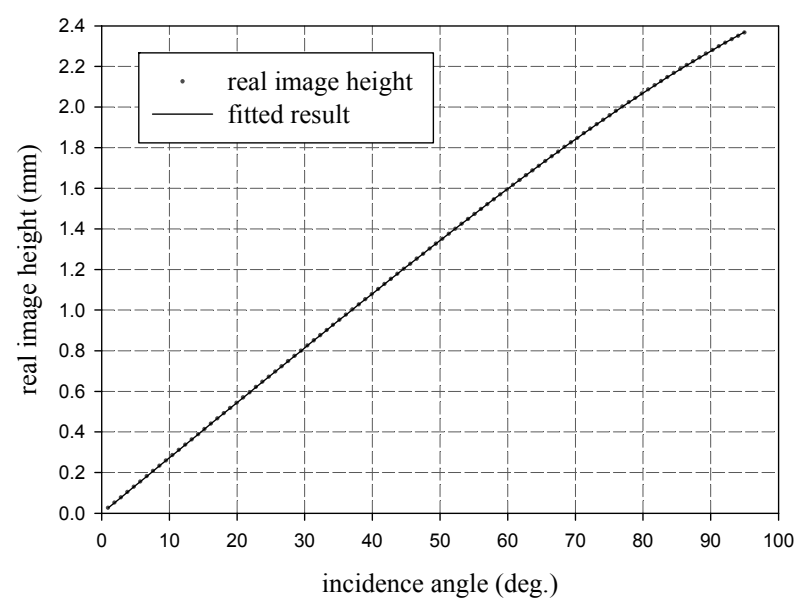

FIG. 20. Real image height for the visible wavelength and a best fit curve using odd powered polynomial terms up to $5^{\text {th }}$ order.

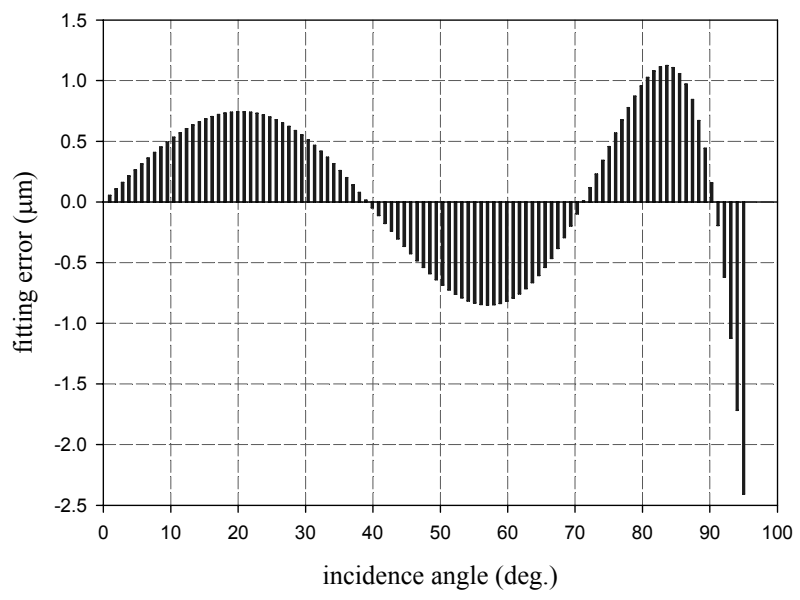

FIG. 21. Remnant error in Fig. 20.
TABLE 2. Fitting coefficients of the real image height for the visible wavelength using odd powered polynomial terms up to $5^{\text {th }}$ order.

\begin{tabular}{c|c}
\hline \hline coefficient & value \\
\hline $\mathrm{a}$ & 1.561590 \\
\hline $\mathrm{b}$ & $-2.289045 \times 10^{-2}$ \\
\hline $\mathrm{c}$ & $-9.257548 \times 10^{-3}$ \\
\hline
\end{tabular}

TABLE 3. Fitting coefficients of the real image height for the visible wavelength using both odd and even powered polynomial terms up to $5^{\text {th }}$ order.

\begin{tabular}{c|c}
\hline \hline coefficient & value \\
\hline $\mathrm{a}$ & 1.560778 \\
\hline $\mathrm{b}$ & $3.054932 \times 10^{-2}$ \\
\hline $\mathrm{c}$ & $-1.078742 \times 10^{-1}$ \\
\hline $\mathrm{d}$ & $7.612269 \times 10^{-2}$ \\
\hline $\mathrm{e}$ & $-3.101406 \times 10^{-2}$ \\
\hline
\end{tabular}

VGA-grade $(640 \times 480$ pixels $) \quad 1 / 3$-inch image sensor has square pixels with each side measuring $7.5 \mathrm{\mu m}$. Therefore, the discrepancy can be as large as two pixels. For security camera equipped with infrared illuminator, visible wavelength light will be dominant during the day time, and near infrared wavelength light will be dominant during the night time. Advanced security cameras are equipped with mechanically changeable optical filters. In consequence, infrared is effectively blocked during the day time where average illumination is high. On the other hand, in low light conditions, the optical filter is switched so that the infrared wavelength is allowed to reach the image sensor. For demanding applications, separate image processing coefficients can be used for the day time and the night time.

Figure 20 shows the real projection scheme (dotted line) of the lens in the visible wavelength range and the best polynomial fit (solid line) to the real projection scheme using odd powered polynomial terms given in Eq. (3). Here, the unit of the image height is in millimeters and the unit of incidence angle $\theta$ is in radians.

$$
r_{\text {odd }}(\theta)=a \theta+b \theta^{3}+c \theta^{5}
$$

The real image heights read from the merit function have been fitted to a polynomial passing through the origin using MatLab. The fitting coefficients are given in table 2. As can be seen from Fig. 20, the real projection scheme can be well approximated by a polynomial curve passing through the origin. Figure 21 shows the remnant error between the real projection scheme and the best polynomial fit, and the maximum error is less than 2.5 


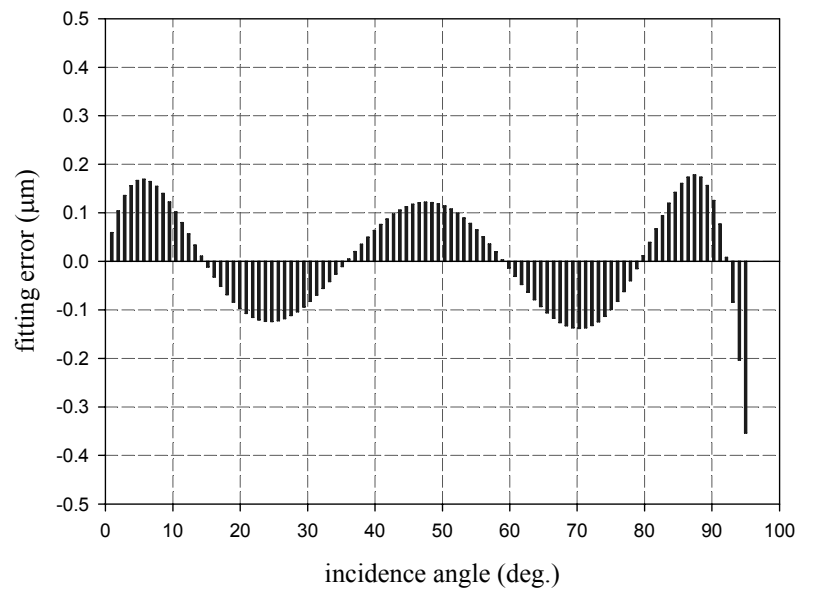

FIG. 22. Reduced fitting error using both odd and even powered polynomial terms up to $5^{\text {th }}$ order term.

TABLE 4. Fitting coefficients of the real image height for the near infrared wavelength using odd powered polynomial terms up to $5^{\text {th }}$ order.

\begin{tabular}{c|c}
\hline \hline coefficient & value \\
\hline $\mathrm{a}$ & 1.571609 \\
\hline $\mathrm{b}$ & $-2.424787 \times 10^{-2}$ \\
\hline $\mathrm{c}$ & $-8.887674 \times 10^{-3}$ \\
\hline
\end{tabular}

TABLE 5. Fitting coefficients of the real image height for the near infrared wavelength using both odd and even powered polynomial terms up to $5^{\text {th }}$ order.

\begin{tabular}{c|c}
\hline \hline coefficient & value \\
\hline $\mathrm{a}$ & 1.570828 \\
\hline $\mathrm{b}$ & $3.006187 \times 10^{-2}$ \\
\hline $\mathrm{c}$ & $-1.079893 \times 10^{-1}$ \\
\hline $\mathrm{d}$ & $7.504669 \times 10^{-2}$ \\
\hline $\mathrm{e}$ & $-3.034208 \times 10^{-2}$ \\
\hline
\end{tabular}

$\mu \mathrm{m}$. Considering typical pixel sizes, the fit is already good enough for most applications.

Figure 22 shows the remnant error between the real projection scheme and the best polynomial fit to the curve using both odd and even powered polynomial terms given in Eq. (4).

$$
r_{p o l}(\theta)=a \theta+b \theta^{2}+c \theta^{3}+d \theta^{4}+e \theta^{5} .
$$

By employing both odd and even powered terms, the discrepancy could be reduced below $0.5 \mu \mathrm{m}$. Similar fitting results for infrared wavelength range are given in tables 4 and 5, respectively.

\section{CONCLUSION}

In conclusion, we have designed and fabricated a F2.8 fisheye lens with a FOV of $190^{\circ}$ operating simultaneously in the visible and the near infrared wavelengths range. The real projection schemes of the lens have been accurately characterized so that this lens can be employed in image processing applications without an error-prone experimental determination stage.

\section{ACKNOWLEDGMENT}

This work was supported by the Regional Technology Innovation Program (BPF-2007-R07) of the Ministry of Knowledge Economy(MKE). We would like to thank director Ilmok Kim at Siwon Optical Technology for his enthusiastic assistance in design and manufacturing of the fisheye lens.

\section{REFERENCES}

[1] R. W. Wood, "Fish-eye views, and vision under water," Phil. Mag. S. 6, vol. 12, no. 68, pp. 159-162, 1906.

[2] W. N. Bond, "A wide angle lens for cloud recording," Phil. Mag. S. 6, vol. 44, no. 263, pp. 999-1001, 1922.

[3] R. Hill, “A lens for whole sky photographs," $Q$. J. R. Meteor. Soc., vol. 50, pp. 227-235, 1924.

[4] C. Beck, "Apparatus to photograph the whole sky," J. Sci. Instrum., vol. 2, pp. 135-139, 1925.

[5] J. M. Slater, "Photography with the whole-sky lens," Am. Photographer, pp. 580-583, 1932.

[6] K. Miyamoto, "Fish-eye lens," J. Opt. Soc. Am., vol. 54, pp. 1060-1061, 1964.

[7] M. Isshiki and K. Matsuki, "Achromatic super wideangle lens,” US patent 3524697, 1970.

[8] Y. Shimizu, "Wide-angle fisheye lens," US patent 3737214, 1973.

[9] R. Doshi, "Fisheye projection lens for large format film,” Proc. SPIE, vol. 2000, pp. 53-61, 1993.

[10] T. Weigel, B. Moll, and B. J. Beers, "Ghost image debugging on a 240 degree fisheye lens," Proc. SPIE, vol. 2774, pp. 598-609, 1996.

[11] J. J. Kumler and M. Bauer, "Fisheye lens designs and their relative performance," Proc. SPIE, vol. 4093, pp. 360-369, 2000.

[12] K. Yasuhiro and Y. Kazuyoshi, "Fisheye lens and photographing apparatus with the same," Japanese patent 2006-098942, 2006.

[13] A. Ning, "Compact fisheye objective lens," US patent 7023628, 2006.

[14] M. Kawada, “Fisheye lens unit," US patent 7283312, 2007.

[15] N. Alvertos, E. L. Hall, and R. L. Anderson, "Omnidirectional viewing for robot vision," Proceedings of the SPIE conference on robot vision and sensory control (Cambridge, MA, Nov. 6-10, 1983), pp. 309-318.

[16] M. Ehtashami, S. J. Oh, and E. L. Hall, "Omnidirec- 
tional position location for mobile robots," Proc. SPIE, vol. 521, pp. 62-73, 1984.

[17] S. D. Zimmermann, "Omniview motionless camera orientation system,” US patent 5185667, 1993.

[18] H. L. Martin and D. P. Kuban, "System for omnidirectional image viewing at a remote location without the transmission of control signals to select viewing parameters," US patent 5384588, 1995.

[19] E. Gullichsen and S. Wyshynski, "Wide-angle image dewarping method and apparatus,” US patent 6005611, 1999.

[20] N. L. Max, "Computer graphics distortion for IMAX and OMNIMAX projection," Proc. NICOGRAPH'83, pp. 137-159, 1983.

[21] N. Greene, "Environment mapping and other applications of world projections," IEEE Computer Graphics and Applications, vol. 6, no. 11, pp. 21-29, 1986.

[22] T. J. Herbert, "Calibration of fisheye lenses by inversion of area projections," Appl. Opt., vol. 25, no. 12, pp. 1875-1876, 1986.

[23] Z. Zhang, "A flexible new technique for camera calibration,” IEEE Transactions on Pattern Analysis and Machine Intelligence, vol. 22, no. 11, pp. 1330-1334, 2000 . 